International Journal of Pure and Applied Mathematics

Volume 92 No. 4 2014, 535-547

ISSN: 1311-8080 (printed version); ISSN: 1314-3395 (on-line version)

url: http://www.ijpam.eu

doi: http://dx.doi.org/10.12732/ijpam.v92i4.8

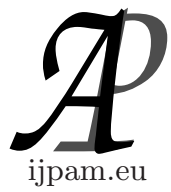

\title{
BERNOULLI'S n-FORMULA AND n_MULTI-SERIES BY THE GENERALIZED DIFFERENCE EQUATION
}

\author{
G. Britto Antony Xavier ${ }^{1}$, H. Nasira Begum ${ }^{2}$, B. Govindan ${ }^{3}$ \\ ${ }^{1,2,3}$ Department of Mathematics \\ Sacred Heart College \\ Tirupattur, 635601, Vellore District, Tamil Nadu, S. INDIA
}

\begin{abstract}
We derive the Bernoulli's n-Formula and n_multi-series by equating the numerical and complete solution of the $n^{\text {th }}$ order generalized difference equation. We also illustrate 2_multi-series to product of arithmetic and geometric functions.
\end{abstract}

AMS Subject Classification: 39A70, 47B39, 39A10

Key Words: complete solution, Bernoulli's series, difference operator, generalized difference equation, numerical solution

\section{Introduction}

The Fractional Calculus is currently a very important research field in several different areas: physics (including classical and quantum mechanics and thermodynamics), chemistry, biology, economics and control theory ([5], [6], [7]). In 1989, K.S.Miller and Ross [8] introduced the discrete analogue of the Riemann-Liouville fractional derivative and proved some properties of the fractional difference operator. The main definition of fractional difference equation (as done in [8]) is the $\nu$ fractional sum of $f(t)$ by

$$
\Delta^{-\nu} f(t)=\frac{1}{\Gamma_{(\nu)}} \sum_{s=a}^{t-\nu} \frac{\Gamma_{(t-s)}}{\Gamma_{(t-s-(\nu-1))}} f(s),
$$

where $\nu>0$. On the other hand, when $\nu=m$ is a positive integer, if we replace

Received: December 23, 2013

(c) 2014 Academic Publications, Ltd.

$\S$ Correspondence author url: www.acadpubl.eu 
the function $f(t)$ by $u(k)$ and $\Delta$ by $\Delta_{\ell}$ defined as $\Delta_{\ell} u(k)=u(k+\ell)-u(k),(1)$ becomes

$$
\mathrm{u}_{n(\ell)}(k)=\Delta_{\ell}^{-n} u(k)=\sum_{r=n}^{\left[\frac{k}{\ell}\right]} \frac{(r-1)^{(n-1)}}{(n-1) !} u(k-r \ell) .
$$

Let $\ell_{i}>0, u(k)$ be real valued function on $[0, \infty), u(k)=0$ for all $k \in$ $(-\infty, 0],\left[k / \ell_{i}\right]$ be the integer part of $k / \ell_{i}, \ell_{i}(k)=k-\left[k / \ell_{i}\right] \ell_{i}$ for $i=1,2, \cdots n$, $\ell_{o}(k)=k$ and $r^{(n)}=r(r-1) \cdots(r-(n-1))$. Then, for $n \geq 2$, (2) induces $n \_$multi-series

$$
\mathrm{u}_{[1, n]}(k)=\sum_{r_{n}=1}^{\left[\frac{k}{\ell_{n}}\right]} \underset{\ell_{[1, n-1]}}{\mathrm{u}}\left(k-r_{n} \ell_{n}\right),
$$

where $\underset{\ell_{[1,1]}}{\mathrm{u}}(k)=\sum_{r_{1}=1}^{\left[\frac{k}{\ell_{1}}\right]} u\left(k-r_{1} \ell_{1}\right)\left(1\right.$ _series with respect to $\left.\ell_{1}\right)$,

$\underset{\ell_{[1,2]}}{\mathrm{u}}(k)=\sum_{r_{2}=1}^{\left[\frac{k}{\ell_{2}}\right]} \ell_{[1,1]} \mathrm{u}\left(k-r_{2} \ell_{2}\right)\left(2 \_\right.$multi-series with respect to $\left.\ell_{1}, \ell_{2}\right)$ and in general

$\mathrm{U}_{[1, i]}(k)=\sum_{r_{i}=1}^{\left[\frac{k}{\ell_{i}}\right]} \mathrm{\ell}_{[1, i-1]} \mathrm{u}\left(k-r_{i} \ell_{i}\right)\left(\mathrm{i}_{-}\right.$multi-series w.r.to $\left.\ell_{1}, \ell_{2}, \cdots, \ell_{n}\right)$.

Substituting $\underset{\ell_{[1,1]}}{\mathrm{u}}, \underset{\ell_{[1,2]}}{\mathrm{u}}, \cdots, \underset{\ell_{[1, n-1]}}{\mathrm{u}}$ in (3), we get

$$
\mathrm{u}(k)=\sum_{r_{n}=1}^{\left[\frac{k}{\ell_{n}}\right]} \sum_{r_{n-1}=1}^{\left[\frac{k-r_{n} \ell_{n}}{\ell_{n}-1}\right]} \sum_{r_{n-2}=1}^{\left[\frac{k-r_{n} \ell_{n}-r_{n-1} \ell_{n-1}}{\ell_{n-2}}\right]} \cdots \sum_{r_{1}=1}^{\left[\frac{k-\sum_{i=2}^{n} r_{i} \ell_{i}}{\ell_{1}}\right]} u\left(k-\sum_{i=1}^{n} r_{i} \ell_{i}\right),
$$

which is a numerical solution of the generalized difference equation

$$
\underset{\ell_{[1, n]}}{\Delta} v(k)=\Delta_{\ell_{1}}\left(\Delta_{\ell_{2}} \cdots \Delta_{\ell_{n}}(v(k)) \cdots\right)=u(k), \quad k \geq 0 .
$$

By denoting R.H.S of (4) as $\sum_{\ell_{[1, n]}} u(\tilde{k}),(3)$ and (4) become

$$
\mathrm{\ell}_{[1, n]}^{\mathrm{u}}(k)=\sum_{\ell_{[1, n]}} u(\tilde{k}) .
$$

In particular, when $n=\underset{\ell_{[1,1]}}{1, \mathrm{u}(\mathrm{k})}={\left.\underset{\ell_{[1,1]}}{\Delta^{-1}} u(k)\right|_{\ell_{1}(k)} ^{k}}_{k}^{k} u(\tilde{k})$. 
Remark 1.1. $\sum_{\ell_{[m, n]} \ell_{[1, m-1]}} \mathrm{u}\left(\ell_{m-1}(\tilde{k})\right)$ denotes

$$
\left.\sum_{r_{n}=1}^{\left[\frac{k}{\ell_{n}}\right]} \sum_{r_{n-1}=1}^{\left[\frac{k-r_{n} \ell_{n}}{\ell_{n}-1}\right.} \cdots \sum_{r_{m}=1}^{\left[\frac{k-\sum_{i=m+1}^{n} r_{i} \ell_{i}}{\ell_{m}}\right.}\right] \underset{\ell_{[1, m-1]}}{\mathrm{u}}\left(\ell_{m-1}\left(k-\sum_{i=m}^{n} r_{i} \ell_{i}\right)\right) .
$$

When $\ell_{1}=\ell_{2}=\cdots \ell_{n}=\ell$, the above $n \_$multi-series $\sum_{\ell_{[1, n]}} u(\tilde{k})$ becomes $\underset{n(\ell)}{u_{(}}(k)$ given in $(2)$. We find that, by expanding the terms, $\underset{\ell_{[1, n]}}{\mathrm{u}}(k)$ is independent of the order of the parameters $\ell_{1}, \ell_{2}, \cdots, \ell_{n}$. There are direct formulas to find the $n_{-}$series when $u(k)=k^{m}, k_{\ell}^{(m)}, a^{k}, k^{m} a^{k}$ etc and $\ell_{1}=\ell_{2}=\cdots=\ell_{n}=\ell$ $[3,4]$.

There is no direct formula to find the sum of $n \_$multi-series in the existing literature. We find that the $n \_$multi-series $\sum_{\ell_{[1, n]}} u(\tilde{k})$ is the numerical solution as well as the complete solution (closed form solution with lower limits) of equation (5), so we call $\mathrm{u}_{\ell_{[1, n]}}(k)$ as the complete solution and $\sum_{\ell_{[1, n]}} u(\tilde{k})$ as the numerical solution of (5). Hence in this paper, we obtain the numerical-complete relation (6) and derive n_multi-series with Bernoulli's n-formula.

\section{Preliminaries}

In this section, we present some notations, basic definitions and preliminary results. Let $J_{n}=\{1,2, \ldots, n\}, 0\left(J_{n}\right)=\{\phi\}, \phi$ is the empty set, $1\left(J_{n}\right)=$ $\{\{1\},\{2\}, \cdots,\{n\}\}, 2\left(J_{n}\right)=\{\{1,2\},\{1,3\}, \cdots,\{1, n\},\{2,3\}, \cdots,\{2, n\}$, $\cdots,\{n-2, n-1\}\}$. In general, $t\left(J_{n}\right)$ is the set of all subsets of size $t$ in ascending order from the set $J_{n}, \wp\left(J_{n}\right)=\bigcup_{t=0}^{n} t\left(J_{n}\right)$ is the power set of $J_{n}, \sum_{t=1}^{n} f(t)=0$ for $n<1, \prod_{i=2}^{t} f(i)=1$ if $t \leq 1, \underset{\ell_{[p, q]}}{\Delta^{-1}} u(k)=\Delta_{\ell_{p}}^{-1}\left(\Delta_{\ell_{p+1}}^{-1} \cdots \Delta_{\ell_{q}}^{-1}(u(k)) \cdots\right)$ for $1 \leq p<q \leq n, \underset{\ell_{[1,0]}}{\mathrm{u}}=u(k), \underset{\ell_{[1,1]}}{\mathrm{u}}(k)=\Delta_{\ell_{1}}^{-1} u(k)$ and for $2 \leq i \leq n, \underset{\ell_{[1, i]}}{\mathrm{u}}(k)=$ $\Delta_{\ell_{i}}^{-1}\left(\left.\underset{\ell_{[1, i-1]}}{\mathrm{u}}(k)\right|_{\ell_{i-1}(k)} ^{k}\right)=\Delta_{\ell_{i}}^{-1} \underset{\ell_{[1, i-1]}}{\mathrm{u}}(k)-\Delta_{\ell_{i}}^{-1} \underset{\ell_{[1, i-1]}}{\mathrm{u}}\left(\ell_{i-1}(k)\right)$.

Lemma 2.1. [2] If $s_{r}^{n}$ and $S_{r}^{n}$ are the Stirling numbers of the first and 
second kinds respectively, $k_{\ell}^{(n)}=k(k-\ell) \cdots(k-(n-1) \ell)$, then

$$
\begin{aligned}
& k_{\ell}^{(n)}=\sum_{r=1}^{n} s_{r}^{n} \ell^{n-r} k^{r} \text { and } k^{n}=\sum_{r=1}^{n} S_{r}^{n} \ell^{n-r} k_{\ell}^{(r)} . \\
& \Delta_{\ell} k_{\ell}^{(n)}=(n \ell) k_{\ell}^{(n-1)} \text { and } \Delta_{\ell}^{-1} k_{\ell}^{(\nu)}=\frac{k_{\ell}^{(\nu+1)}}{(\nu+1) \ell} .
\end{aligned}
$$

Theorem 2.2. [1] (Discrete Bernoulli's Formula)

Let $u(k)$ and $v(k)$ be two real valued functions, $u^{(t)}(k)=\Delta_{\ell}^{t} u(k), v_{t}(k)=$ $\Delta_{\ell}^{-t} v(k)$ for $t=1,2, \cdots$ and $u^{(0)}(k)=u(k)$. Then,

$$
\Delta_{\ell}^{-1}[u(k) v(k)]=\sum_{t=0}^{\infty}(-1)^{t} u^{(t)}(k) v_{t+1}(k+t \ell) .
$$

\section{Main Results And Applications}

Here, by introducing Striling numbers of third kind and expressing the polynomial factorial $k_{\ell_{a}}^{(n)}$ interms of $k_{\ell_{b}}^{(r)}, r=1,2, \cdots n$ and Stirling numbers of third kind, we derive the Bernoulli's n-formula and n_multi-series to product of arithmetic and geometric functions with examples.

Definition 3.1. Let $1 \leq p \leq n$, The Stirling number of third kind for the pair of positive reals $\ell_{a}$ and $\ell_{b}$ is defined by

$$
S_{p \_\ell_{a}}^{n \ell_{b}}=\sum_{t=p}^{n} s_{t}^{n} S_{p}^{t} \ell_{a}^{n-t} \ell_{b}^{t-p}
$$

Lemma 3.2. The expression of $k_{\ell_{a}}^{(n)}$ interms of $k_{\ell_{b}}^{(p)}$ is given by

$$
k_{\ell_{a}}^{(n)}=\sum_{p=1}^{n} S_{p_{-} \ell_{a}}^{n_{-} \ell_{b}} k_{\ell_{b}}^{(p)}
$$

Proof. The proof follows from (11) and first, second terms of (8).

Example 3.3. Since $S_{1_{-}}^{5_{-} 4}=0, S_{2_{-}}^{5_{-} 4}=0, S_{3_{-} 2}^{5_{-} 4}=60, S_{4_{-}}^{5_{-}}=20$ and $S_{5_{-2}}^{5_{-4}}=1$, (12) yields $k_{2}^{(5)}=\left(60 \times k_{4}^{(3)}\right)+\left(20 \times k_{4}^{(4)}\right)+\left(1 \times k_{4}^{(5)}\right)$. 
Lemma 3.4. Let $p_{1}=1, \ell_{1}, \ell_{2}, \cdots \ell_{n}$ be a set of positive reals and ${ }_{\ell_{[1, n]}}^{-1}=\Delta_{\ell_{1}}^{-1} \Delta_{\ell_{2}}^{-1} \cdots \Delta_{\ell_{n}}^{-1}$. Then, $\Delta_{\ell_{1}}^{-1} k^{(0)}=\frac{k_{\ell_{1}}^{(1)}}{\ell_{1}}$ and

$$
{\overrightarrow{\ell_{[1, n]}}}^{-1} k^{(0)}=\left[\prod_{r=2}^{n-1} \sum_{p_{r}=1}^{1+p_{r-1}} \frac{S_{p_{r} \ell_{r}}^{1+p_{r-1} \ell_{r+1}}}{\left(1+p_{r-1}\right) \ell_{r}} \mid \frac{k_{\ell_{n}}^{\left(1+p_{n-1}\right)}}{\ell_{1}\left(1+p_{n-1}\right) \ell_{n}} .\right.
$$

Proof. Since $1=k_{\ell_{1}}^{(0)}=k^{(0)}$ and $k_{\ell_{1}}^{(1)}=k_{\ell_{2}}^{(1)}$ from second relation of (9), we get $\Delta_{\ell_{1}}^{-1} k_{\ell_{1}}^{(0)}=\frac{k_{\ell_{1}}^{(1)}}{\ell_{1}}$. Taking $\Delta_{\ell_{2}}^{-1}$, we get $\Delta_{\ell_{[1,2]}}^{-1} k^{(0)}=\frac{k_{\ell_{2}}^{(2)}}{2 \ell_{1} \ell_{2}}$. Again taking $\Delta_{\ell_{3}}^{-1}$ on both sides of the above and applying (12), we obtain $\Delta_{\ell_{[1,3]}^{-1}}^{-1} k^{(0)}=\frac{1}{2 \ell_{1} \ell_{2}} \Delta_{\ell_{3}}^{-1} k_{\ell_{2}}^{(2)}=$ $\sum_{p_{2}=1}^{2} S_{p_{2} \ell_{2}}^{2 \_\ell_{3}} \frac{k_{\ell_{3}}^{\left(1+p_{2}\right)}}{2 \ell_{1} \ell_{2} \ell_{3}\left(1+p_{2}\right)}$

Now the proof is completed by taking $\Delta_{\ell_{i}}^{-1}$ and applying second relation of (9) and (12) for $i=4,5, \cdots, n$ respectively.

Lemma 3.5. Let $p_{0} \in N(0)$ and $S_{p_{-} \ell_{a}}^{n_{-} \ell_{b}}$ be the Stiriling number of third kind. Then,

$$
{\overrightarrow{\ell_{[1, n]}}}^{-1} k_{\ell_{1}}^{\left(p_{o}\right)}=\prod_{r=1}^{n-1} \sum_{p_{r}=1}^{1+p_{r-1}} \frac{S_{p_{r} \ell_{r}}^{1+p_{r-1} \ell_{r+1}}}{\left(1+p_{r-1}\right) \ell_{r}} \frac{k_{\ell_{n}}^{\left(1+p_{n-1}\right)}}{\left(1+p_{n-1}\right) \ell_{n}}
$$

Proof. Taking $\nu=p_{o}$ and $\ell=\ell_{1}$ in second relation of (9), we get

$$
\Delta_{\ell_{1}}^{-1} k_{\ell_{1}}^{\left(p_{o}\right)}=\frac{k_{\ell_{1}}^{\left(p_{o}+1\right)}}{\left(p_{o}+1\right) \ell_{1}} .
$$

The proof follows by repeatedly applying (12), $\Delta_{\ell_{i}}^{-1}$ and second relation of (9) for $i=2,3, \cdots, n$.

Theorem 3.6. A closed form solution of (5) for $u(k)=k^{p_{o}}$ is

$$
{\overrightarrow{\left.\ell^{\prime}, n\right]}}^{-1} k^{p_{o}}=\prod_{r=2}^{n} \sum_{p_{r}=1}^{1+p_{r-1}} \sum_{p_{1}=1}^{p_{o}} S_{p_{1}}^{p_{o}} \ell_{1}^{p_{o}-p_{1}} \frac{S_{p_{r} \ell_{r-1}}^{1+p_{r-1} \ell_{r}}}{\left(1+p_{r-1}\right) \ell_{r-1}} \frac{k_{\ell_{n}}^{\left(1+p_{n}\right)}}{\left(1+p_{n}\right) \ell_{n}} .
$$


Proof. Replacing $\mathrm{n}$ by $p_{o}, \mathrm{r}$ by $p_{1}$ and $\ell$ by $\ell_{1}$ in second relation of (8), we obtain $k^{p_{o}}=\sum_{p_{1}=1}^{p_{o}} S_{p_{1}}^{p_{o}} \ell_{1}^{p_{o}-p_{1}} k_{\ell_{1}}^{\left(p_{1}\right)}$.

Applying $\Delta_{\ell_{1}}^{-1}$ and using second relation of (9), we get

$$
\Delta_{\ell_{1}}^{-1} k^{p_{o}}=\sum_{p_{1}=1}^{p_{o}} S_{p_{1}}^{p_{0}} \ell_{1}^{p_{o}-p_{1}} \frac{k_{\ell_{1}}^{\left(1+p_{1}\right)}}{\left(1+p_{1}\right) \ell_{1}}
$$

Using (12) in (16), we obtain

$$
\Delta_{\ell_{1}}^{-1} k^{p_{o}}=\sum_{p_{1}=1}^{p_{o}} S_{p_{1}}^{p_{o}} \ell_{1}^{p_{o}-p_{1}} \sum_{p_{2}=1}^{1+p_{1}} S_{p_{2_{-} \ell_{1}}}^{\ell_{1+p_{1}}{ }^{\ell_{2}}} \frac{k_{\ell_{2}}^{\left(p_{2}\right)}}{\left(1+p_{1}\right) \ell_{1}}
$$

The proof of (15) follows by contuining this process $\mathrm{n}$ times,

The following Theorem is the generalization of Theorem 2.2.

Theorem 3.7. (DISCRETE BERNOULLI'S n-FORMULA)

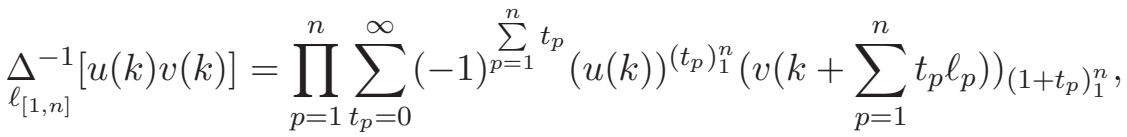

$$
\begin{aligned}
& \text { where }(u(k))^{\left(t_{p}\right)_{1}^{n}}=\Delta_{\ell_{1}}^{t_{1}}\left(\cdots\left(\Delta_{\ell_{n}}^{t_{n}} u(k)\right) \cdots\right),\left(v\left(k+\sum_{p=1}^{n} t_{p} \ell_{p}\right)\right)_{\left(1+t_{n}\right)_{1}^{n}} \\
& =\Delta_{\ell_{1}}^{-\left(1+t_{1}\right)}\left(\Delta_{\ell_{2}}^{-\left(1+t_{2}\right)} \cdots\left(\Delta_{\ell_{n}}^{-\left(1+t_{n}\right)} v\left(k+\sum_{p=1}^{n} t_{p} \ell_{p}\right) \cdots\right)\right) \text { and } \\
& \prod_{p=1}^{n} \sum_{t_{p}=0}^{\infty}=\sum_{t_{1}=0}^{\infty} \sum_{t_{2}=0}^{\infty} \cdots \sum_{t_{n}=0}^{\infty} \text {. }
\end{aligned}
$$

Proof. From Theorem 2.2, we have

$$
\Delta_{\ell_{1}}^{-1}[u(k) v(k)]=\sum_{t_{1}=0}^{\infty}(-1)^{t_{1}}(u(k))^{\left(t_{1}\right)}\left(v\left(k+t_{1} \ell_{1}\right)\right)_{\left(1+t_{1}\right)} .
$$

Taking $\Delta_{\ell_{2}}^{-1}$ on both sides, we get

$$
\underset{\ell_{[1,2]}}{\Delta^{-1}}[u(k) v(k)]=\prod_{p=1}^{2} \sum_{t_{p}=0}^{\infty}(-1)^{t_{1}+t_{2}}(u(k))^{\left(t_{p}\right)_{1}^{2}}\left(v\left(k+t_{1} \ell_{1}+t_{2} \ell_{2}\right)\right)_{\left(1+t_{p}\right)_{1}^{p} .}
$$

The proof follows by taking $\Delta_{\ell_{i}}^{-1}$, for $i=3,4, \cdots, n$ successively on both sides of (18). 
Lemma 3.8. Let $t_{i}^{\prime} s$ and $p_{o}$ are positive integers. Then,

$$
\prod_{i=1}^{n} \Delta_{\ell_{i}}^{t_{i}}\left(k_{\ell_{1}}^{\left(p_{o}\right)}\right)=p_{o}^{\left(t_{1}\right)} \ell_{1}^{t_{1}} \prod_{r=1}^{n-1} \sum_{p_{r}=1}^{p_{r-1}-t_{r}} p_{r}^{t_{r+1}} \ell_{r+1}^{t_{r+1}} S_{p_{r} \ell_{r}}^{p_{r-1}-t_{r} \ell_{r+1}} k_{\ell_{n}}^{\left(p_{n-1}-t_{n}\right)}
$$

Proof. From first relation of (9), we have

$$
\Delta_{\ell_{1}}^{t_{1}}\left(k_{\ell_{1}}^{\left(p_{o}\right)}\right)=p_{o}^{\left(t_{1}\right)} \ell_{1}^{t_{1}} k_{\ell_{1}}^{\left(p_{o}-t_{1}\right)}
$$

Taking $\Delta_{\ell_{2}}^{t_{2}}$ on both sides of the above equation, we obtain

$$
\Delta_{\ell_{2}}^{t_{2}}\left(\Delta_{\ell_{1}}^{t_{1}}\left(k_{\ell_{1}}^{\left(p_{o}\right)}\right)\right)=p_{o}^{\left(t_{1}\right)} \ell_{1}^{t_{1}} \Delta_{\ell_{2}}^{t_{2}}\left(k_{\ell_{1}}^{\left(p_{o}-t_{1}\right)}\right) .
$$

Applying (12), we get

$$
\Delta_{\ell_{2}}^{t_{2}}\left(\Delta_{\ell_{1}}^{t_{1}}\left(k_{\ell_{1}}^{\left(p_{o}\right)}\right)\right)=p_{o}^{\left(t_{1}\right)} \ell_{1}^{t_{1}} \Delta_{\ell_{2}}^{t_{2}}\left(\sum_{p_{1}=1}^{p_{o}-t_{1}} S_{p_{1} \ell_{1}}^{p_{p_{1}}-t_{1} \ell_{2}} k_{\ell_{2}}^{\left(p_{1}\right)}\right) .
$$

The proof follows by repeating this process $\mathrm{n}$ times.

Lemma 3.9. If $a^{\ell_{i}}-1 \neq 0$, for $i=1,2, \cdots n$, then

$$
\Delta_{\ell_{1}}^{-\left(1+t_{1}\right)}\left(\Delta_{\ell_{2}}^{-\left(1+t_{2}\right)}\left(\cdots \Delta_{\ell_{n}}^{-\left(1+t_{n}\right)} a^{k}\right)\right)=\frac{a^{k}}{\prod_{i=1}^{n}\left(a^{\ell_{i}}-1\right)^{\left(1+t_{i}\right)}} .
$$

Proof. Since $\Delta_{\ell} a^{k}=a^{k+\ell}-a^{k}=a^{k}\left(a^{\ell}-1\right)$, we have

$$
\Delta_{\ell_{1}}^{-\left(1+t_{1}\right)} a^{k}=\frac{a^{k}}{\left(a^{\ell_{1}}-1\right)^{\left(1+t_{1}\right)}} .
$$

Taking $\Delta_{\ell_{2}}^{-\left(1+t_{2}\right)}$ on both sides of the above equation, we get

$$
\Delta_{\ell_{1}}^{-\left(1+t_{1}\right)}\left(\Delta_{\ell_{2}}^{-\left(1+t_{2}\right)} a^{k}\right)=\frac{a^{k}}{\left(a^{\ell_{1}}-1\right)^{\left(1+t_{1}\right)}\left(a^{\ell_{2}}-1\right)^{\left(1+t_{2}\right)}} .
$$

The proof follows by repeatedly taking $\Delta_{\ell_{i}}^{-\left(1+t_{i}\right)}$ on both sides for $i=3,4, \cdots, n$.

Theorem 3.10. Bernoulli's $n_{-}$Formula to the product $k_{\ell}^{\left(p_{o}\right)} a^{k}$ is

$$
{\overrightarrow{\left.\ell^{\prime}, n\right]}}^{-1}\left(k_{\ell_{1}}^{\left(p_{o}\right)} a^{k}\right)=\prod_{p=1}^{n} \sum_{t_{p}=0}^{p_{o}}(-1)^{\sum_{p=1}^{n} t_{p}}\left(k_{\ell_{1}}^{\left(p_{o}\right)}\right)^{\left(t_{p}\right)_{1}^{n}}\left(a^{\left(k+\sum_{p=1}^{n} t_{p} \ell_{p}\right)}\right)_{\left(1+t_{p}\right)_{1}^{n} .}
$$


Proof. Since $\left(k_{\ell_{1}}^{\left(p_{o}\right)}\right)^{\left(t_{1}\right)\left(t_{2}\right) \cdots\left(t_{n}\right)}=0$ when $t_{1}+t_{2}+\cdots+t_{n}>p_{o}$ and $t_{1}, t_{2}, \cdots, t_{n}$ take the values from 0 to $p_{0}$, we have

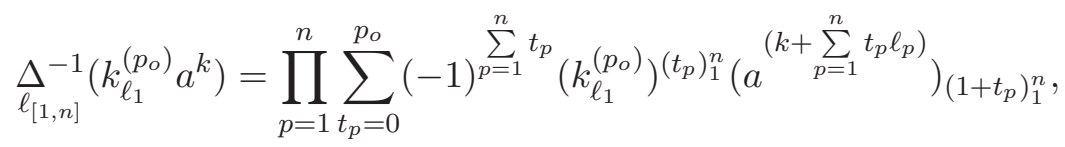

where $\left(k_{\ell_{1}}^{\left(p_{o}\right)}\right)^{\left(t_{p}\right)_{1}^{n}}=\left(k_{\ell_{1}}^{\left(p_{o}\right)}\right)^{\left(t_{1}\right)\left(t_{2}\right) \cdots\left(t_{n}\right)}=\left\{\begin{array}{l}\Delta_{\ell_{1}}^{t_{1}}\left(\Delta_{\ell_{2}}^{t_{2}}\left(\cdots \Delta_{\ell_{n}}^{t_{n}} k_{\ell_{1}}^{\left(p_{o}\right)}\right)\right) \\ 0 \text { if } t_{1}, t_{2}, \cdots, t_{n}>p_{o},\end{array}\right.$

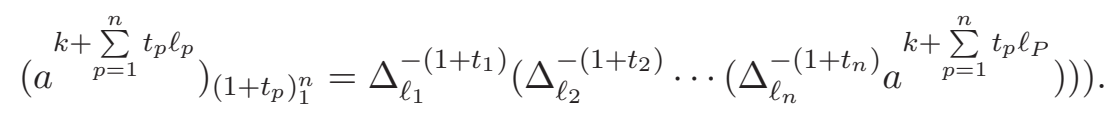

(21) follows by taking $u(k)=k_{\ell}^{\left(p_{o}\right)}$ and $v(k)=a^{k}$ in (17).

The following theorem gives complete solution of equation (5).

Theorem 3.11. Consider the functions $\underset{\ell_{[1, i]}}{\mathrm{u}}(k), \ell_{i}(k)$ for $i=1,2, \cdots, n$, given in the notations and above. Assume that for each $i, 1 \leq i \leq n, \Delta_{\ell_{[1, i]}}^{-1} u(k)$ be any closed form solution of the difference equation $\underset{\ell_{[1, i]}}{\Delta} v(k)=u(k)$. Then, for $k \geq \max _{1 \leq i \leq n} \ell_{i}$,

$$
\begin{aligned}
& \left.\mathrm{u}_{\ell_{[1, n]}}(k)\right|_{\ell_{n}(k)} ^{k}=\Delta_{\ell_{[1, n]}}^{-1} u(k)+\sum_{t=1}^{n}(-1)^{t} \sum_{\left\{m_{s}\right\}_{s=1}^{t} \in t\left(J_{n}\right)} \Delta_{\left[1, m_{1}\right]}^{\Delta^{-1}} u\left(\ell_{m_{1}}(k)\right) \times \\
& \times \prod_{i=1}^{t} \underset{\ell_{\left[1+m_{i}, m_{i+1}\right]}}{\Delta^{-1}}\left(\ell_{m_{i+1}}(k)\right)^{(0)} \underset{\ell_{\left[1+m_{t}, n\right]}}{\Delta^{-1}} k^{(0)}
\end{aligned}
$$

is the complete solution of equation (5).

Proof. Since $1=k^{(0)}$, applying the limit from $\ell_{1}(k)$ to $k$ for $\Delta_{\ell_{1}}^{-1} u(k)$, we have $\left.\Delta_{\ell_{1}}^{-1} u(k)\right|_{\ell_{1}(k)} ^{k}=\Delta_{\ell_{1}}^{-1} u(k)-\Delta_{\ell_{1}}^{-1} u\left(\ell_{1}(k)\right) k^{(0)}$, which is the complete solution of equation (5) for $n=1$.

Taking $\Delta_{\ell_{2}}^{-1}$ on both sides and applying the limits from $\ell_{2}(k)$ to $k$ and keeping $\Delta_{\ell_{1}}^{-1} u\left(\ell_{1}(k)\right)$ as a constant, we obtain

$$
\left.\Delta_{\ell_{2}}^{-1}\left(\left.\Delta_{\ell_{1}}^{-1} u(k)\right|_{\ell_{1}(k)} ^{k}\right)\right|_{\ell_{2}(k)} ^{k}=\left.\Delta_{\ell_{[1,2]}}^{-1} u(k)\right|_{\ell_{2}(k)} ^{k}-\left.\Delta_{\ell_{1}}^{-1} u\left(\ell_{1}(k)\right) \Delta_{\ell_{2}}^{-1} k^{(0)}\right|_{\ell_{2}(k)} ^{k}
$$

which is the complete solution of the equation (5), and it can be expressed as

$$
\left.\underset{\ell_{[1,2]}}{\mathrm{u}}(k)\right|_{\ell_{2}(k)} ^{k}=\Delta_{\ell_{[1,2]}}^{-1} u(k)-\Delta_{\ell_{1}}^{-1} u\left(\ell_{1}(k)\right) \Delta_{\ell_{2}}^{-1} k^{(0)}
$$




$$
-\Delta_{\ell_{[1,2]}}^{-1} u\left(\ell_{2}(k)\right)+\Delta_{\ell_{1}}^{-1} u\left(\ell_{1}(k)\right) \Delta_{\ell_{2}}^{-1}\left(\ell_{2}(k)\right)^{(0)} .
$$

In the R.H.S of the above expression, second term is associated to $\left\{m_{1}\right\}=\{1\} \in$ $1\left(J_{2}\right)$, third term to $\left\{m_{1}\right\}=\{2\} \in 1\left(J_{2}\right)$ and the fourth term to $\left\{m_{1}, m_{2}\right\}=$ $\{1,2\} \in 2\left(J_{2}\right)$. Taking $\Delta_{\ell_{3}}^{-1}$ on $u_{2}(k)$, applying the limits $\ell_{3}(k)$ and $k$, and as $\Delta_{\ell_{1}}^{-1} u\left(\ell_{1}(k)\right), \Delta_{\ell_{2}}^{-1}\left(\ell_{2}(k)\right)^{(0)}$ and $\Delta_{\ell_{[1,2]}}^{-1} u\left(\ell_{2}(k)\right)$ are constants, we get a relation of the form $\left.\operatorname{u}_{\ell_{[1,3]}}(k)\right|_{\ell_{3}(k)} ^{k}=\Delta_{\ell_{3}}^{-1} \operatorname{l}_{[1,2]}^{u}(k)-\Delta_{\ell_{3}}^{-1} \underset{\ell_{[1,2]}}{u}\left(\ell_{3}(k)\right)$, and this relation can be expressed as

$$
\begin{aligned}
\left.\operatorname{\ell }_{[1,3]}^{\mathrm{u}}(k)\right|_{\ell_{3}(k)} ^{k} & =\Delta_{\ell_{[1,3]}}^{-1} u(k)+\sum_{t=1}^{3} \sum_{\left\{m_{s}\right\}_{1}^{t} \in t\left(J_{n}\right)}(-1)^{t}{\underset{\ell_{\left[1, m_{1}\right]}}{\Delta^{-1}} u\left(\ell_{m_{1}}(k)\right)} \times \prod_{i=1}^{t} \underset{\ell_{\left[1+m_{i}, m_{i+1}\right]}}{\Delta^{-1}}\left(\ell_{m_{i+1}}(k)\right)^{(0)} \underset{\ell_{\left[1+m_{t}, 3\right]}^{\Delta^{-1}} k^{(0)},}{ }
\end{aligned}
$$

which is a complete solution of the equation (5) for $n=3$.

As all the lower limit values are constants, the proof is completed by taking $\Delta_{\ell_{i}}^{-1}$ and applying the limit from $\ell_{i}(k)$ to $k$ on $\mathrm{u}_{[1,3]}(k)$ successively for $i=$ $4,5, \cdots, n$.

The following theorem gives the numerical solution of the equation (5).

Theorem 3.12. Consider the terms of Theorem 3.11. Then,

$$
v(k)=\sum_{m=1}^{n} \sum_{\ell_{[m, n]}} \underset{\ell_{[1, m-1]}}{\mathrm{u}}\left(\ell_{m-1}(\tilde{k})\right), \quad k \geq \sum_{i=1}^{n} \ell_{i}
$$

is the numerical solution of the difference equation (5).

Proof. From equation (7), we have

$$
\left.\Delta_{\ell_{1}}^{-1} u(k)\right|_{\ell_{1}(k)} ^{k}=\sum_{\ell_{[1,1]}} u(\tilde{k})=\operatorname{u}_{\ell_{[1,1]}}(k)-\operatorname{u}_{\ell_{[1,1]}}^{\mathrm{u}}\left(\ell_{1}(k)\right)=z_{1}(k), \text { (say) }
$$

is a numerical solution of the equation (5) for $n=1$. Again taking $\Delta_{\ell_{2}}^{-1}$ on $z_{1}(k)$ and applying equation (7), we get

$$
\left.\Delta_{\ell_{2}}^{-1} z_{1}(k)\right|_{\ell_{2}(k)} ^{k}=\sum_{\ell_{[2,2]}} z_{1}(\tilde{k})=z_{2}(k), \text { (say) }
$$


which is a numerical solution of the equation (5) for $n=2$.

Replacing $k$ by $k-r_{2} \ell_{2}$ in (26), we obtain

$$
z_{1}\left(k-r_{2} \ell_{2}\right)=\mathrm{uu}_{\ell_{[1,1]}}\left(k-r_{2} \ell_{2}\right)-\underset{\ell_{[1,1]}}{\mathrm{u}}\left(\ell_{1}\left(k-r_{2} \ell_{2}\right)\right) .
$$

Substituting (28) in (27), we find that

$$
z_{2}(k)=\sum_{\ell_{[2,2]}} \mathrm{u}_{[1,1]}(\tilde{k})-\sum_{\ell_{[2,2]}} \mathrm{u}_{[1,1]}\left(\ell_{1}(\tilde{k})\right)
$$

which is the same as

$$
z_{2}(k)=\mathrm{u}_{\ell_{[1,2]}}(k)-\mathrm{u}_{\ell_{[1,2]}}\left(\ell_{2}(k)\right)-\sum_{\ell_{[2,2]}} \mathrm{\ell}_{[1,1]}\left(\ell_{1}(\tilde{k})\right) .
$$

Applying the numerical solution $z_{2}(k)=\sum_{\ell_{[1,2]}} u(\tilde{k})$ on $(30)$, we get

$$
\left.\mathrm{u}_{\ell_{[1,2]}}(k)\right|_{\ell_{2}(k)} ^{k}=\sum_{\ell_{[1,2]}} u(\tilde{k})+\sum_{\ell_{[2,2]}} \mathrm{u}_{[1,1]}\left(\ell_{1}(\tilde{k})\right)
$$

where the values $\mathrm{u}_{\ell_{[1,1]}}\left(\ell_{1}\left(k-r_{2} \ell_{2}\right)\right)$ can be evaluated by replacing $k$ by $\ell_{1}\left(k-r_{2} \ell_{2}\right)$ in the complete solution $\left.\underset{\ell_{[1,1]}}{\mathrm{u}}(k)\right|_{\ell_{1}(k)} ^{k}$ given in Theorem 3.11 for $n=1$.

The proof is completed by taking $\Delta_{\ell_{i}}^{-1}$ on $z_{2}(k)$ and applying the numerical solution of equation (5) successively for $i=3,4,5, \cdots, n$.

Theorem 3.13. The numerical-complete relation of the equation (5) is given by

$$
\begin{aligned}
\sum_{m=1}^{n} \sum_{\ell_{[m, n]}} \ell_{[1, m-1]} \mathrm{u}\left(\ell_{m-1}(\tilde{k})\right)=\Delta_{\ell_{[1, n]}}^{-1} u(k)+\sum_{t=1}^{n} \sum_{\left\{m_{s}\right\}_{s=1}^{t} \in t\left(J_{n}\right)}(-1)^{t} \times \\
\quad \times \Delta_{\ell_{\left[1, m_{1}\right]}}^{\Delta^{-1}} u\left(\ell_{m_{1}}(k)\right) \prod_{i=1}^{t} \underset{\ell_{\left[1+m_{i}, m_{i+1}\right]}}{\Delta^{-1}}\left(\ell_{m_{i+1}}(k)\right)^{(0)} \underset{\ell_{\left[1+m_{t}, n\right]}}{\Delta^{-1}} k^{(0)} .
\end{aligned}
$$

Proof. The proof follows by equating the numerical solution given in Theorem 3.12 and the complete solution given in Theorem 3.11.

Theorem 3.14. $n \_$multi-series to the polynomial factorial $k_{\ell_{1}}^{p_{o}}$ is

$$
\sum_{m=1}^{n} \sum_{\ell_{[m, n]}} \mathrm{u}_{\ell_{[1, m-1]}}\left(\ell_{m-1}(\tilde{k})\right)=\Delta_{\ell_{[1, n]}}^{-1} k_{\ell_{1}}^{\left(p_{o}\right)}+\sum_{t=1}^{n} \sum_{\left\{m_{s}\right\}_{s=1}^{t} \in t\left(J_{n}\right)}(-1)^{t} \times
$$




$$
\times \underset{\ell_{\left[1, m_{1}\right]}}{\Delta^{-1}}\left(\ell_{m_{1}}(k)\right)_{\ell_{1}}^{\left(p_{o}\right)} \prod_{i=1}^{t} \underset{\ell_{\left[1+m_{i}, m_{i+1}\right]}}{\Delta^{-1}}\left(\ell_{m_{i+1}}(k)\right)^{(0)} \underset{\ell_{\left[1+m_{t}, n\right]}}{\Delta^{-1}} k^{(0)} .
$$

Proof. The proof follows by taking $u(k)=k_{\ell_{1}}^{\left(p_{o}\right)}$ in $(32)$ and the terms in (33) are evaluated by (13) and (14).

Theorem 3.15. n_multi-series formula to the polynomial $k^{p_{o}}$ is

$$
\begin{aligned}
& \sum_{m=1}^{n} \sum_{\ell_{[m, n]}} \mathrm{\ell}_{[1, m-1]} \mathrm{u}\left(\ell_{m-1}(\tilde{k})\right)=\Delta_{\ell_{[1, n]}}^{-1} k^{p_{o}}+\sum_{t=1}^{n} \sum_{\left\{m_{s}\right\}_{s=1}^{t} \in t\left(J_{n}\right)}(-1)^{t} \times \\
& \quad \times \Delta_{\ell_{\left[1, m_{1}\right]}^{-1}}^{\Delta^{-1}}\left(\ell_{m_{1}}(k)\right)^{p_{o}} \prod_{i=1}^{t} \underset{\ell_{\left[1+m_{i}, m_{i+1}\right]}}{\Delta^{-1}}\left(\ell_{m_{i+1}}(k)\right)^{(0)} \underset{\ell_{\left[1+m_{t}, n\right]} \Delta^{-1} k^{(0)} .}{ }
\end{aligned}
$$

Proof. The proof follows by taking $u(k)=k^{p_{o}}$ in (32) and the terms in (34) are evaluated by (15).

Theorem 3.16. n_multi-series formula to $U(k)=u(k) v(k)$ is

$$
\begin{aligned}
& \sum_{m=1}^{n} \sum_{\ell_{[m, n]} \ell_{[1, m-1]}} \mathrm{U}\left(\ell_{m-1}(\tilde{k})\right)=\Delta_{\ell_{[1, n]}^{-1}}(u(k) v(k))+\sum_{t=1}^{n} \sum_{\left\{m_{s}\right\}_{s=1}^{t} \in t\left(J_{n}\right)}(-1)^{t} \\
& \quad \times \Delta_{\ell_{\left[1, m_{1}\right]}}^{-1} u\left(\ell_{m_{1}}(k)\right) v\left(\ell_{m_{1}}(k)\right) \prod_{i=1}^{t} \underset{\ell_{\left[1+m_{i}, m_{i+1}\right]}}{\Delta^{-1}}\left(\ell_{m_{i+1}}(k)\right)^{(0)} \underset{\ell_{\left[1+m_{t}, n\right]} \Delta^{-1} k^{(0)} .}{ }
\end{aligned}
$$

Proof. The proof follows by taking $U(k)=u(k) v(k)$ in Theorem 3.13 and $\Delta_{\ell_{[1, n]}}^{\Delta^{-1}}[u(k) v(k)]$ can be obtain by Bernoulli's n-Formula .

The following example illustrates Therom 3.16.

Example 3.17. Taking $n=2, u(k)=k_{\ell_{1}}^{(2)}=(u(k))^{(0)(0)}, v(k)=a^{k}$ in (35) and using (17), we get

$$
(u(k))^{\left(t_{p}\right)_{1}^{2}}=\Delta_{\ell_{1}}^{t_{1}}\left(\Delta_{\ell_{2}}^{t_{2}} u(k)\right)=\Delta_{\ell_{1}}^{t_{1}}\left(\Delta_{\ell_{2}}^{t_{2}} k_{\ell_{1}}^{(2)}\right) .
$$

From $(36),(u(k))^{(0)(1)}=2 \ell_{2} k_{\ell_{2}}^{(1)}+\left(\ell_{2}-\ell_{1}\right) \ell_{2},(u(k))^{(0)(2)}=2 \ell_{2}^{2}$, $(u(k))^{(1)(0)}=2 \ell_{1} k_{\ell_{2}}^{(1)},(u(k))^{(1)(1)}=2 \ell_{1} \ell_{2},(u(k))^{(2)(0)}=2 \ell_{1}^{2}$ and

$$
(v(k))_{\left(1+t_{p}\right)_{1}^{2}}=\Delta_{\ell_{1}}^{-\left(1+t_{1}\right)}\left(\Delta_{\ell_{2}}^{-\left(1+t_{2}\right)} a^{k}\right)=\frac{a^{k}}{\prod_{i=1}^{2}\left(a^{\ell_{i}}-1\right)^{1+t_{i}}} .
$$


(37) yields $(v(k))_{(1)(1)},(v(k))_{(1)(2)},(v(k))_{(1)(3)},(v(k))_{(2)(1)},(v(k))_{(2)(2)},(v(k))_{(2)(3)}$, $(v(k))_{(3)(1)}$ and $(v(k))_{(3)(2)}$.

To find the corresponding (35), consider:

(i) $\Delta_{\ell_{[1,2]}}^{-1}\left(k_{\ell_{1}}^{(2)} a^{k}\right)=\frac{k_{\ell_{2}}^{(2)} a^{k}}{\left(a^{\ell_{1}}-1\right)\left(a^{\ell_{2}}-1\right)}-\frac{2 \ell_{2} k_{\ell_{2}}^{(1)} a^{k+\ell_{2}}}{\left(a^{\ell_{1}}-1\right)\left(a^{\ell_{2}}-1\right)^{2}}$

$$
\begin{aligned}
& +\frac{2 \ell_{2}^{2} a^{k+2 \ell_{2}}}{\left(a^{\ell_{1}}-1\right)\left(a^{\ell_{2}}-1\right)^{3}}+\left\{\frac{\left(\ell_{2}-\ell_{1}\right)}{\left(a^{\ell_{1}}-1\right)}-\frac{2 \ell_{1} a^{\ell_{1}}}{\left(a^{\ell_{1}}-1\right)^{2}}\right\} \\
& \times\left\{\frac{k_{\ell_{2}}^{(1)} a^{k}}{\left(a^{\ell_{2}}-1\right)}-\frac{\ell_{2} a^{k+\ell_{2}}}{\left(a^{\ell_{2}}-1\right)^{2}}\right\}+\frac{2 \ell_{1}^{2} a^{k+2 \ell_{1}}}{\left(a^{\ell_{1}}-1\right)^{3}\left(a^{\ell_{2}}-1\right)} .
\end{aligned}
$$

(ii) the terms for $1\left(J_{2}\right)=\{\{1\},\{2\}\}$ :

$(-1) \Delta_{\ell_{[1,1]}}^{-1}\left(\ell_{1}(k)\right)_{\ell_{1}}^{(2)} a^{\ell_{1}(k)} \Delta_{\ell_{[2,2]}}^{-1} k^{(0)} \quad$ and $(-1) \Delta_{\ell_{[1,2]}}^{-1}\left(\ell_{2}(k)\right)_{\ell_{1}}^{(2)} a^{\ell_{2}(k)}$.

(iii) the terms for $2\left(J_{2}\right)=\{1,2\}: \Delta_{\ell_{[1,1]}}^{-1}\left(\ell_{1}(k)\right)_{\ell_{1}}^{(2)} a^{\ell_{1}(k)} \Delta_{\ell_{[2,2]}}^{-1}\left(\ell_{2}(k)\right)_{\ell_{2}}^{(0)}$.

In particular taking $k=5, \ell_{1}=1, \ell_{2}=2, \ell_{1}(k)=0, \ell_{2}(k)=1$ and $a=2$ in (35), we get (i) 37.92592 (ii) 20 and 13.92592 (iii) 4 .

R.H.S of (35) is the sum of the terms $(i)+(i i)+(i i i)=8$.

L.H.S of (35) is 8 , which is obtained by adding the terms:

$m=1 ; \quad \sum_{\ell_{[1,2]}} u(\tilde{k})=0$ and $m=2 ; \quad \sum_{\ell_{[2,2]}} \ell_{[1,1]}^{\mathrm{u}}\left(\ell_{1}(\tilde{k})\right)=8$.

Conclusion: Finding the complete solution of higher order Generalized difference equations coincided with the numerical solution of that equation is the significant of the research work. This relation generates certain formulas and some applications for finding the growth of animal population of first $n$ generations.

\section{Acknowledgments}

Research Supported by University Grants Commission, SERO, Hyderabad, India.

\section{References}

[1] G.Britto Antony Xavier and H.Nasira Begum, Discrete Bernoulli's Formula and its Applications arising from Generalized Difference Operator, International Journal of Mathematical Analysis, 7 (5) (2013), 229-240.

[2] M.Maria Susai Manuel, G.Britto Antony Xavier and E.Thandapani, The- 
ory of Generalized Difference Operator and Its Applications, Far East Journal of Mathematical Sciences, 20(2) (2006), 163 - 171.

[3] M.Maria Susai Manuel, G.Britto Antony Xavier and E.Thandapani, Generalized Bernoulli Polynomials Through Weighted Pochhammer Symbols, Far East Journal of Applied Mathematics, 26(3) (2007), 321-333.

[4] M.Maria Susai Manuel, V.Chandrasekar and G.Britto Antony Xavier, Some Applications of the Generalized Difference Operator of the $n^{\text {th }}$ Kind, Far East Journal of Applied Mathematics, 66(2) (2012), 107 - 126.

[5] A.A.Kilbas, H.M.Srivastava and J.J.Trujillo, Theory and application of fractional differential equations, Elsevier, Amsterdam, 2006.

[6] K.S.Miller,B.Ross, An introduction to the fractional calculus and fractional differential equations, Wiley, New york, 1993.

[7] S.G.Samko, A.A.Kibas and O.I.Marichev, Fractional integrals and derivatives, Translated from the 1987 Russian original, Gordon and Breach, Yverdon, 1993.

[8] K.S.Miller,B.Ross , Fractional difference calculus, in "Univalent functions, fractional calculus, and the applications (Koriyama, 1988)", 139-152, Horwood, Chichester, 1989. 
